\title{
MODELING THE MECHANICAL RESPONSE OF A MATERIAL UNDERGOING CONTINUOUS ISOTHERMAL CRYSTALLIZATION
}

\author{
MEHRDAD NEGAHBAN \\ Department of Engineering Mechanics, University of Nebraska-Lincoln, Lincoln, \\ NE 68588-0347, U.S.A.
}

ALAN S. WINEMAN

Department of Mechanical Engineering and Applied Mechanics, University of Michigan, Ann Arbor, MI 48109, U.S.A.

\begin{abstract}
The gradual transition seen in polymer crystallization is modeled. A constitutive equation is developed to follow the mechanical behavior of a crystallizing polymer before, during, and after the completion of crystallization. The post-crystallization response of the material is studied and shown to be "elastic". The symmetries of the post-crystallization response are defined and calculated for crystallization under several deformation histories.
\end{abstract}

\section{INTRODUCTION}

Phase transitions, such as crystallization, are gradual and continuous in most polymers $[1,2]$. This paper is concerned with modeling these gradual transitions. In particular, we will develop a constitutive equation which can be used to follow the mechanical behavior during and after the completion of crystallization.

It is important to note that time, in addition to temperature, strain and preconditioning, influences the speed of transformation and the mechanical properties after the completion of the transition [1,3]. For example, the rate of crystallization is, in general, increased by increasing strain [1]. Also, the mechanical properties of the crystallized material, such as stiffness and strength of anisotropy, depend on the type of strain imposed in the crystallization process $[4,5]$.

Since the conditions of crystallization (i.e. time, strain and temperature) strongly influence the mechanical response of the material at each stage of the transition, the constitutive equation should also reflect this dependence. In this paper we present an isothermal model with the following features:

(1) The model can follow the mechanical response of a material through the three stages of its transition. These three stages are:

(a) the response of the amorphous material before the crystallization process starts;

(b) the response during the transition from the amorphous to the amorphouscrystalline state;

(c) the response after the crystallization stops, the post-crystallization response.

(2) The model does not assume the form of the post-crystallization response. The characteristics of this response follow as a consequence of the specific conditions of crystallization (i.e. deformation history during crystallization), and the mathematical structure of the model. As a result, the model can be used to calculate process induced symmetries and the final mechanical properties.

(3) The model is three dimensional and fully nonlinear, both materially and geometrically. Preliminary definitions and basic assumptions as to how the phase transition influences the mechanical response are presented in Section 2. Sections 3 and 4 are devoted to imposing the restrictions of frame-indifference and material symmetry.

The constitutive equation for an initially isotropic material is developed in Section 5. Sections 6,7 and 8 are devoted to studying the post-crystallization response of an initially 
isotropic material. The post-crystallization response is defined in Section 6. Section 7 contains a study of the material symmetries of the post-crystallization response for crystallization under several deformation histories. These results are independent of the change in material parameters in the constitutive equation. Section 8 contains results for a specific choice of material parameters.

\section{CONSTITUTIVE ASSUMPTIONS}

In this section we formulate a constitutive equation which can represent the mechanical response of a material throughout the various stages of the crystallization process. These stages are (a) the response of the amorphous material before the crystallization process starts, (b) the response during the transition from the amorphous to the amorphous-crystalline state, and (c) the response after the crystallization stops.

On a microscopic level, crystallization is the process which takes a material's unorganized amorphous structure into a well organized crystal structure. In many materials, like metals, this transformation is very abrupt and the material "fully" transforms from one state of structure to the other. On the other hand, in polymers this transition is more gradual [1]. In many cases, the process of crystallization in a polymer is stopped long before all the polymer crystallizes [1]. This type of gradual crystallization is the focus of this paper, even though very rapid crystallization can also be modeled by this method.

The constitutive model is developed based on the following assumptions:

Assumption 1. The properties of a particle in a continuum model represent the average, in some sense, of the properties in some small neighborhood of the actual material point being modelled. During the crystallization process, such a neighborhood can contain both amorphous and crystalline matter.

Thus, we assume that there can be particles of both amorphous and crystalline matter at each point of the continuum. This is indicated in Fig. 1.

Assumption 2. We assume that a single continuum defines the material through all stages of its transformation.

Let $\kappa_{0}$ denote the configuration of the material in its initial state and let $\kappa(s)$ denote its configuration at a later time $s$. Let the coordinates of the same typical material particle be

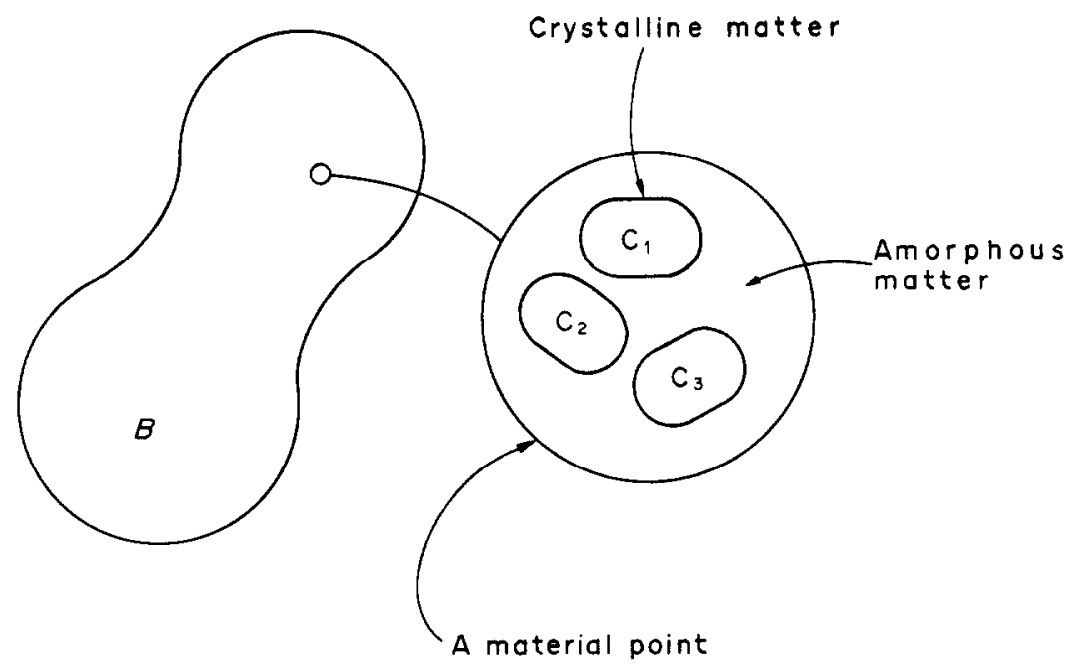

Fig. 1. At each continuum point there can be both amorphous and crystallized matter simultaneously. 
denoted by $\mathbf{X}$ in configuration $\kappa_{o}$ and hy $\mathbf{x}(s)$ in configuration $\kappa(s)$. The deformation gradient comparing $\kappa(s)$ to $\kappa_{o}$ will be denoted by $\mathbf{F}(s)$.

Assumption 3. We assume that at time $t$ the Cauchy stress, $\mathbf{T}(t)$, is the superposition of contributions from the crystalline portions formed up to time $t$ and from the remaining portion of amorphous matter at time $t$. We assume that crystals can be generated continuously and that the response of all crystals generated at the same time can be represented by a single constitutive equation. We also assume that each type of matter contributes to the stress in proportion to its current mass ratio. $\dagger$

An expression for the Cauchy stress which is consistent with this assumption is given by

$$
\mathbf{T}(t)=b(t) \mathbf{T}^{A}(t)+\int_{t_{s}}^{t} a(s) \mathbf{T}^{C}(t, s) \mathrm{d} s
$$

In (1), $b(t)$ denotes the mass ratio of the amorphous material which has not crystallized up to time $t, b(t) \mathbf{T}^{A}(t)$ denotes the contribution of this portion of the material to the value of the stress at time $t, t_{s}$ is the time when crystallization starts, $a(s) \mathrm{d} s$ is the mass ratio of material crystallized in the interval from $s$ to $s+\mathrm{d} s$, and $a(s) \mathbf{T}^{C}(t, s) \mathrm{d} s$ is the contribution of this crystallized material to the value of the stress at time $t$. As can be seen, equation (1) is a special averaging method for obtaining the macroscopic stress of a mixture of one type of amorphous material and many different types of crystals. In this equation time acts as a marker to distinguish between crystals generated at different times and under different conditions.

Conservation of mass requires that the quantities $b(t)$ and $a(s)$ be related by

$$
b(t)=1-\int_{t_{s}}^{t} a(s) \mathrm{d} s
$$

Equation (2) should be accompanied by a relation of the form

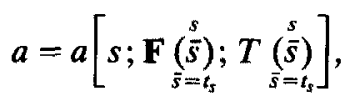

which expresses the rate of crystallization in terms of the deformation history, the temperature history and possibly other influences. A specific relation will not be needed for the purpose of the present study. $\neq$

Assumption 4. It is assumed that the amorphous and crystalline phases of the material each respond elastically.

That is, the stress in the amorphous part is related only to $F(t)$, the gradient comparing $k(t)$ to $\boldsymbol{K}_{0}$. This stress is given as

$$
\mathbf{T}^{A}(t)=F^{(1)}[\mathbf{F}(t)]
$$

where $\mathscr{F}^{(1)}$ is a function of $\mathbf{F}(t)$. The stress in the crystalline part formed during the interval from $s$ to $s+\mathrm{d} s$ depends on $\mathbf{F}(t)$ and also on $\mathbf{F}_{s}(t)$, the gradient comparing $\kappa(t)$ to $\kappa(s)$, the configuration at time $s$ when it was formed. This stress is given as

$$
\mathbf{T}^{C}=\mathscr{F}^{(2)}\left[\mathbf{F}(t) ; \mathbf{F}_{s}(t) ; s\right],
$$

†An alternate model can be developed which would use the current value of the volume ratio. Such a model would require providing added conditions to allow the calculation of the current value of the volume ratio in terms of the past history. In view of the many other simplifications, it is not clear that adding this complexity is justified. An example of such a model is developed Appendix B.

\#Examples of such models can be found in $[3,6,7]$. 
where $\mathscr{F}^{(2)}$ is a function of the stated arguments. Note that $F_{s}(t)$ can be written as $\mathbf{F}_{s}(t)=\mathbf{F}(t) F^{-1}(s)$, and, therefore, the variable set $\mathbf{F}(t)$ and $\mathbf{F}_{s}(t)$ can always be replaced by the variable set $\mathbf{F}(t)$ and $\mathbf{F}(s)$ or the variable set $\mathbf{F}_{s}(t)$ and $\mathbf{F}(s)$. The final form of the constitutive equation is obtained by combining (1), (4) and (5) to give

$$
\mathbf{T}(t)=b(t) \mathscr{F}^{(1)}[\mathbf{F}(t)]+\int_{t_{s}}^{t} a(s) \mathscr{F}^{(2)}\left[\mathbf{F}(t) ; \mathbf{F}_{s}(t) ; s\right] \mathrm{d} s .
$$

Equation (6) can represent the response of the material in each of the three phases discussed above. Before the onset of crystallization $a=0$ in (2) and (6). This results in the usual form of constitutive equation for nonlinear elastic response. When $a \neq 0$, equations (2), (3) and (6) give the response during the transition from amorphous to the amorphous-crystalline state. Finally, when crystallization stops, $a=0$. As will be shown in a later section, when crystallization stops the right hand side of (6) varies only with $\mathbf{F}(t)$. That is, (6) becomes a constitutive equation for an elastic material, but with new response characteristics.

\section{MATERIAL FRAME INDIFFERENCE}

Consider a motion of the material $\mathbf{x}=\mathbf{x}(\mathbf{X}, t)$ whose deformation gradient is $\mathbf{F}(t)$. A second motion which differs from the first by a rigid body motion, can be written as $\mathbf{x}^{*}(t)=$ $\mathbf{Q}(t) \mathbf{x}(\mathbf{X}, t)+\mathbf{c}(t)$ where $\mathbf{Q}(t)$ represents a rigid body rotation (i.e. $\left.\mathbf{Q}(t) \mathbf{Q}^{T}(t)=\mathbf{Q}^{T}(t) \mathbf{Q}(t)=\mathbf{I}\right)$ and $\mathbf{c}(t)$ represents a pure rigid body translation. The deformation gradient of this second motion is $\mathbf{F}^{*}(t)=\mathbf{Q}(t) \mathbf{F}(t)$.

The requirement of material frame indifference is that the response functional on the right hand side of (6) satisfy the condition

$$
\begin{aligned}
\mathbf{Q}(t)\left\{b(t) \mathscr{F}^{(1)}[\mathbf{F}(t)]+\int_{t_{s}}^{t} a(s)\right. & \left.\mathscr{F}^{(2)}\left[\mathbf{F}(t) ; \mathbf{F}_{s}(t) ; s\right] \mathrm{d} s\right\} \mathbf{Q}^{T}(t) \\
& =b(t) \mathscr{F}^{(1)}[\mathbf{Q}(t) \mathbf{F}(t)]+\int_{t_{s}}^{t} a(s) \mathscr{F}^{(2)}\left[\mathbf{Q}(t) \mathbf{F}(t) ; \mathbf{Q}(t) \mathbf{F}_{s}(t) \mathbf{Q}^{T}(s) ; s\right] \mathrm{d} s
\end{aligned}
$$

for all rotations $\mathbf{Q}(s), t_{s} \leq s \leq t . \dagger$

Let the polar decompositions of $\mathbf{F}(t)$ and $\mathbf{F}_{s}(t)$ be written in the form

$$
\mathbf{F}(t)=\mathbf{R}(t) \mathbf{U}(t), \quad \mathbf{F}_{s}(t)=\mathbf{V}_{s}(t) \mathbf{R}_{s}(t),
$$

where $\mathbf{R}(t)$ and $\mathbf{R}_{s}(t)$ represent the orthogonal factors and $\mathbf{U}(t)$ and $\mathbf{V}_{s}(t)$ are symmetric. Let $\mathbf{Q}(s)=\mathbf{R}^{T}(t) \mathbf{R}_{s}(t)$ and note that $\mathbf{Q}(t)=\mathbf{R}^{T}(t)$ since $\mathbf{R}_{t}(t)=\mathbf{I}$. A standard argument then implies that the restriction of material frame indifference will be met if $(6)$ is written in the form

$$
\mathbf{T}(t)=b(t) \mathbf{R}(t) \mathscr{G}^{(1)}[\mathbf{C}(t)] \mathbf{R}^{T}(t)+\int_{t_{s}}^{t} a(s) \mathbf{R}(t)^{\mathscr{G}^{(2)}}\left[\mathbf{C}(t) ; \mathbf{R}(t)^{T} \mathbf{B}_{s}(t) \mathbf{R}(t) ; s\right] \mathbf{R}^{T}(t) \mathrm{d} s .
$$

In (9), $\mathbf{C}(t)=\mathbf{F}^{T}(t) \mathbf{F}(t)=\mathbf{U}^{2}(t), \mathbf{B}_{s}(t)=\mathbf{F}_{s}(t) \mathbf{F}_{s}^{T}(t)=\mathbf{V}_{s}^{2}(t)$, and $\mathscr{G}^{(1)}$ and $\mathscr{G}^{(2)}$ are new functions of the stated arguments.

\section{THE MATERIAL SYMMETRY RESTRICTION}

Let $g_{o}$ denote the symmetry group which is associated with the initial state of the material, and represented with respect to the reference configuration $\kappa_{o}$. Let $\mathbf{H}$ be a symmetry transformation of this group. The material symmetry restriction states that the stress determined by the deformation gradient history $\mathbf{F}(s)$ is the same as would be obtained if the 
material were first subjected to the symmetry transformation $\mathbf{H}$ and then the deformation gradient history $\mathbf{F}(s)$, for every $\mathbf{H} \in g_{o}$. That is, using either $\mathbf{F}(s)$ or $\mathbf{F}(s) \mathbf{H}$ should result in the same value for the Cauchy Stress. Using this in (9) leads to a restriction which can be satisfied if $\mathscr{G}^{(1)}$ and $\mathscr{G}^{(2)}$ are such that

$$
\mathbf{H}^{\left(\mathscr{G}^{(1)}\right.}\left[\mathbf{H}^{T} \mathbf{C}(t) \mathbf{H}\right] \mathbf{H}^{T}=\mathscr{G}^{(1)}[\mathbf{C}(t)]
$$

and

$$
\mathbf{H}^{(2)}\left[\mathbf{H}^{T} \mathbf{C}(t) \mathbf{H} ; \mathbf{H}^{T} \mathbf{R}^{T}(t) \mathbf{B}_{s}(t) \mathbf{R}(t) \mathbf{H} ; s\right] \mathbf{H}^{T}=\mathscr{G}^{(2)}\left[\mathbf{C}(t) ; \mathbf{R}^{T}(t) \mathbf{B}_{s}(t) \mathbf{R}(t) ; s\right]
$$

\section{INITIALLY ISOTROPIC MATERIALS}

In its initial state, the microstructure of the amorphous material consists of randomly oriented and coiled macromolecules. It is thus reasonable to assume that the material is initially isotropic, and that $g_{o}=\mathcal{O} \equiv$ \{full group of orthogonal transformations $\}$. It follows from well-known results for an isotropic tensor function of a single tensor argument that for function (') $^{(1)}$ which satisfies (10),

$$
\mathbf{R}(t) \mathscr{G}^{(1)}[\mathbf{C}(t)] \mathbf{R}^{T}(t)=p_{0} \mathbf{I}+p_{1} \mathbf{B}(t)+p_{2} \mathbf{B}^{2}(t)
$$

where $\mathbf{B}(t)=\mathbf{F}(t) \mathbf{F}^{T}(t)$, and $p_{0}, p_{1}, p_{2}$ are scalar functions of the three isotropic invariants of $\mathbf{B}(t)$.

Rivlin and Erickson [8] have provided a representation for an isotropic tensor function of two tensor arguments. It follows from their results that for the function $\mathscr{G}^{(2)}$ which satisfies (11),

$$
\begin{aligned}
\mathbf{R}(t) \mathscr{G}^{(2)}\left[\mathbf{C}(t) ; \mathbf{R}^{T}(t) \mathbf{B}_{s}(t) \mathbf{R}(t) ; s\right] \mathbf{R}^{T}(t)= & w_{1} \mathbf{I}+w_{2} \mathbf{B}(t)+w_{3} \mathbf{B}^{2}(t)+w_{4} \mathbf{B}_{s}(t)+w_{5} \mathbf{B}_{s}^{2}(t) \\
& +w_{6}\left[\mathbf{B}(t) \mathbf{B}_{s}(t)+\mathbf{B}_{s}(t) \mathbf{B}(t)\right]+w_{7}\left[\mathbf{B}^{2}(t) \mathbf{B}_{s}(t)+\mathbf{B}_{s}(t) \mathbf{B}^{2}(t)\right] \\
& +w_{8}\left[\mathbf{B}(t) \mathbf{B}_{s}^{2}(t)+\mathbf{B}_{s}^{2}(t) \mathbf{B}(t)\right]+w_{9}\left[\mathbf{B}^{2}(t) \mathbf{B}_{s}^{2}(t)+\mathbf{B}_{s}^{2}(t) \mathbf{B}^{2}(t)\right] .
\end{aligned}
$$

Coefficients $w_{1}, w_{2}, \ldots, w_{9}$ are scalar valued functions of the three isotropic invariants of $\mathbf{B}(t)$

$$
I_{1}=\operatorname{tr}(\mathbf{B}(t)), \quad I_{2}=\frac{1}{2}\left[I_{1}^{2}-\operatorname{tr}\left(\mathbf{B}^{2}(t)\right)\right], \quad I_{3}=\operatorname{det}(\mathbf{B}(t))
$$

the three isotropic invariants of $\mathbf{B}_{s}(t)$

$$
I_{4}=\operatorname{tr} \mathbf{B}_{s}(t), \quad I_{5}=\frac{1}{2}\left[I_{4}-\operatorname{tr}\left(\mathbf{B}_{s}^{2}(t)\right)\right], \quad I_{6}=\operatorname{det}\left(\mathbf{B}_{s}(t)\right)
$$

and the four joint invariants of $\mathbf{B}(t)$ and $\mathbf{B}_{s}(t)$

$$
\begin{array}{ll}
I_{7}=\operatorname{tr}\left(\mathbf{B}(t) \mathbf{B}_{s}(t)\right), & I_{8}=\operatorname{tr}\left(\mathbf{B}^{2}(t) \mathbf{B}_{s}(t)\right), \\
I_{9}=\operatorname{tr}\left(\mathbf{B}(t) \mathbf{B}_{s}^{2}(t)\right), & I_{10}=\operatorname{tr}\left(\mathbf{B}^{2}(t) \mathbf{B}_{s}^{2}(t)\right),
\end{array}
$$

where tr stands for the trace operation. On combining (12) and (13) with (9), the constitutive equation becomes

$$
\begin{aligned}
\mathbf{T}(t)= & b(t)\left[p_{0} \mathbf{I}+p_{1} \mathbf{B}(t)+p_{2} \mathbf{B}^{2}(t)\right]+\int_{t_{s}}^{t} a(s)\left\{w_{1} \mathbf{I}+w_{2} \mathbf{B}(t)+w_{3} \mathbf{B}^{2}(t)+w_{4} \mathbf{B}_{s}(t)+w_{5} \mathbf{B}_{s}^{2}(t)\right. \\
& +w_{6}\left[\mathbf{B}(t) \mathbf{B}_{s}(t)+\mathbf{B}_{s}(t) \mathbf{B}(t)\right]+w_{7}\left[\mathbf{B}^{2}(t) \mathbf{B}_{s}(t)+\mathbf{B}_{s}(t) \mathbf{B}^{2}(t)\right] \\
& \left.+w_{8}\left[\mathbf{B}(t) \mathbf{B}_{s}^{2}(t)+\mathbf{B}_{s}^{2}(t) \mathbf{B}(t)\right]+w_{9}\left[\mathbf{B}^{2}(t) \mathbf{B}_{s}^{2}(t)+\mathbf{B}_{s}^{2}(t) \mathbf{B}^{2}(t)\right]\right\} \mathrm{d} s .
\end{aligned}
$$




\section{COMPLETION OF CRYSTALLIZATION-ELASTIC RESPONSE}

Suppose the material starts to crystallize at time $t_{s}$ and continues to crystallize until time $t_{f}$, after which there is no further crystallization. The rate of crystallization function $a(s)$ is then characterized by the conditions

$$
\begin{gathered}
a(s) \neq 0 \text { for some } s \in\left(t_{s}, t_{f}\right], \\
a(s)=0 \text { for all } s \in\left(t_{f}, t\right] .
\end{gathered}
$$

For times $t>t_{f}$, the stress in the material is given by

$$
\begin{aligned}
\mathbf{T}(t)= & b\left(t_{f}\right)\left[p_{0} \mathbf{I}+p_{1} \mathbf{B}(t)+p_{2} \mathbf{B}^{2}(t)\right]+\int_{t_{s}}^{t_{f}} a(s)\left\{w_{1} \mathbf{I}+w_{2} \mathbf{B}(t)+w_{3} \mathbf{B}^{2}(t)+w_{4} \mathbf{B}_{s}(t)\right. \\
& +w_{5} \mathbf{B}_{s}^{2}(t)+w_{6}\left[\mathbf{B}(t) \mathbf{B}_{s}(t)+\mathbf{B}_{s}(t) \mathbf{B}(t)\right]+w_{7}\left[\mathbf{B}^{2}(t) \mathbf{B}_{s}(t)+\mathbf{B}_{s}(t) \mathbf{B}^{2}(t)\right] \\
& \left.+w_{8}\left[\mathbf{B}(t) \mathbf{B}_{s}^{2}(t)+\mathbf{B}_{s}^{2}(t) \mathbf{B}(t)\right]+w_{9}\left[\mathbf{B}^{2}(t) \mathbf{B}_{s}^{2}(t)+\mathbf{B}_{s}^{2}(t) \mathbf{B}^{2}(t)\right]\right\} \mathrm{d} s,
\end{aligned}
$$

where the upper limit of the integral has become fixed at $t_{f}$.

Since in (19) the integration over $s$ stops at $t_{f}$, the only element of the history after $t_{f}$ which contributes to the stress at time $t$ is $\mathbf{F}(t)$. This can be seen if one substitutes for $\mathbf{B}_{s}(t)$ its equivalent expression $\mathbf{B}_{s}(t)=\mathbf{F}(t) \mathbf{C}^{-1}(s) \mathbf{F}^{T}(t)$ into (19), where $\mathbf{C}(s)=\mathbf{F}^{T}(s) \mathbf{F}(s)$. This allows the integral in (19) to be expressed in terms of $\mathbf{F}(t)$ and $\mathbf{C}(s)$, for $s \leq t_{f}$. After the completion of crystallization, the history of events before time $t_{f}$ becomes part of the past history, and, as such, can no longer be considered variable (i.e. we can not change the past). Therefore, after $t_{f}$ the only true variable in the response equation is $\mathbf{F}(t)$.

In other words, after the completion of crystallization one can rewrite (19) in the form

$$
\mathbf{T}(t)=\mathscr{F}[\mathbf{F}(t)]
$$

where $\mathscr{F}$ is a new function. Since for times $t>t_{f}$ the stress depends only on the current value of the deformation gradient, the response can be regarded as being "elastic". The right hand side of $(20)$ will be referred to as the post-crystallization elastic response function. The remainder of this paper will be concerned with the properties of this response function.

It must be noted that, even though the history preceding $t_{f}$ is not explicitly noted in (20), this history is included in the mathematical structure of the response function $\mathscr{F}$ and hence influences its properties. This history can be used to calculate the symmetries of the final elastic response and, also, can be used to evaluate the material constants.

\section{SYMMETRY PROPERTIES OF THE POST-CRYSTALLIZATION ELASTIC RESPONSE FUNCTION}

The material response in the post-crystallization regime is not, in general, isotropic. In order to see this, we will first define the material symmetry properties in this regime, and then discuss how these properties can be determined.

The material symmetries of the post-crystallization response function are defined as those linear transformations $\mathbf{H}$ which satisfy the condition

$$
\mathbf{T}(t)=\mathscr{F}[\mathbf{F}(t)]=\mathscr{F}[\mathbf{F}(t) \mathbf{H}]
$$

identically for any deformation gradient $\mathbf{F}(t)+$ It is straight forward to show that the transformations which satisfy (21) form a group. We will denote this group by $g\left(t_{f}\right)$, and refer to it as the symmetry group at time $t_{f}$. This group represents the symmetries of the material 
after $t_{f}$, but is represented with respect to the original reference configuration $\kappa_{0}$. The explicit form of this group is a result of the material's characteristics and the history of deformations the material has undergone during the crystallization process.

This definition of symmetry is motivated by the following considerations. Suppose a sample of an initially amorphous material crystallizes while it is being deformed. Assume the crystallization has ceased. Now consider an observer attempting to evaluate the symmetries of this sample without being aware of the crystallization process. For this observer, the past history becomes an unknown fixed parameter in the post-crystallization response function of the material. As a result, the observer can only be concerned with the symmetry properties of the response function by considering deformation gradients which occur after the completion of crystallization.

Let us proceed to identify some of the possible symmetries of the post-crystallization response. Let $\mathbf{F}(t)$ be replaced by $\mathbf{F}^{*}(t)=\mathbf{F}(t) \mathbf{H}$. Then $\mathbf{B}(t)$ and $\mathbf{B}_{s}(t)$ become, respectively,

$$
\mathbf{B}^{*}(t)=\mathbf{F}(t) \mathbf{H} \mathbf{H}^{T} \mathbf{F}^{T}(t), \quad \mathbf{B}_{s}^{*}(t)=\mathbf{F}(t) \mathbf{H} \mathbf{C}^{-1}(s) \mathbf{H}^{T} \mathbf{F}^{T}(t) .
$$

Note that $\mathbf{C}(s)$ is left unaltered since $s<t_{f}$ and $t>t_{f} \dagger$

The problem of determining the symmetries of the material is the problem of determining those transformations $\mathbf{H}$ which satisfy (21). This problem is very complicated and specific results can only be obtained under specific sets of assumptions. Two sets of results are presented here.

The first set of results is independent of the form of the scalar coefficients $p_{0}, p_{1}, p_{2}, w_{1}, \ldots, w_{9}$. That is, these results are material independent. In this case, the group of symmetry transformations which satisfy $(21)$ is characterized as follows:

$$
g\left(t_{f}\right) \supseteq\left\{\mathbf{H} \mid \mathbf{H} \in \mathcal{O} \text { and } \mathbf{H}^{T} \mathbf{C}(s) \mathbf{H}=\mathbf{C}(s) \text { for every } s \in\left(t_{s}, t_{f}\right]\right\} .
$$

The " $\supseteq$ " in (23) refers to the fact that for particular forms of the scalar coefficients $p_{0}, p_{1}, p_{2}, w_{1}, \ldots, w_{9}$, and the history of $\mathbf{C}(s)$ for $s \in\left(t_{s}, t_{f}\right]$, there might be other choices of $\mathbf{H}$ which also satisfy (21). The proof of (23) follows from equation (22) since $\mathbf{B}^{*}(t)=\mathbf{B}(t)$ and $\mathbf{B}_{s}^{*}(t)=\mathbf{B}_{s}(t)$ for $\mathbf{H}^{T} \mathbf{H}=\mathbf{I}$ and $\mathbf{H}^{T} \mathbf{C}(s) \mathbf{H}=\mathbf{C}(s)$. This will result in the automatic satisfaction of condition (21). Therefore, as a first step, the problem of finding those $\mathbf{H}$ which satisfy (21) is reduced to the problem of finding those linear orthogonal transformations for which

$$
\mathbf{H}^{T} \mathbf{C}(s) \mathbf{H}=\mathbf{C}(s)
$$

for every $s \in\left(t_{s}, t_{f}\right]$. We now consider several examples for which solutions can be obtained.

\subsection{Crystallization under equal-triaxial extension}

Let the deformation during crystallization be equal-triaxial extension. That is,

$$
\mathbf{C}(s)=\left(\begin{array}{ccc}
\lambda^{2}(s) & 0 & 0 \\
0 & \lambda^{2}(s) & 0 \\
0 & 0 & \lambda^{2}(s)
\end{array}\right)=\lambda^{2}(s) \mathbf{I}
$$

where the stretch ratio $\lambda(s)$ is an arbitrary function of $s$ during the interval $s \in\left(t_{s}, t_{f}\right]$.

When this $\mathbf{C}(s)$ is substituted into (24), the result is

$$
\mathbf{H}^{T} \lambda^{2}(s) \mathbf{I H}=\lambda^{2}(s) \mathbf{I} .
$$

This is satisfied by any transformation $\mathbf{H}$ which satisfies

$$
\mathbf{H}^{T} \mathbf{H}=\mathbf{I},
$$

and therefore

$$
g\left(t_{f}\right) \supseteq 0 .
$$


In other words, when crystallization occurs under an equal-triaxial extension history, the post-crystallization material response will always be an isotropic elastic one.

\subsection{Crystallization under uniaxial (equal-biaxial) extension}

Suppose that the material is subjected to uniaxial extension along a fixed axis during the process of crystallization. Let the third axis of the coordinate system be along the material direction of extension, then

$$
\mathbf{C}(s)=\left(\begin{array}{ccc}
\lambda^{\prime 2}(s) & 0 & 0 \\
0 & \lambda^{\prime 2}(s) & 0 \\
0 & 0 & \lambda^{2}(s)
\end{array}\right),
$$

in which the stretch ratios $\lambda^{\prime}(s)$ and $\lambda(s)$ are arbitrary functions of $s$ and $\lambda(s) \neq \lambda^{\prime}(s)$ for some subinterval of $\left(t_{s}, t_{f}\right]$.

As is shown in [9], the orthogonal transformations $\mathbf{H}$ which satisfy (24) form the group representing transverse isotropy with the preferred axis of transverse isotropy being along the third axis of the material. If this group is denoted as $D_{\text {tran }}$, then

$$
g\left(t_{f}\right) \supseteq D_{\text {tran }} .
$$

Therefore, after crystallization under the history of deformations given by (29), the material response will be at least transversely isotropic, with the preferred axis of transverse isotropy along the axis of the distinct stretch ratio.

\subsection{Crystallization under unequal-triaxial extensions}

Consider a material subjected to unequal-triaxial extensions along a fixed set of material axes during the interval of crystallization. If the axes of extension coincide with the coordinate axes, then

$$
\mathbf{C}(s)=\left(\begin{array}{ccc}
\lambda_{1}^{2}(s) & 0 & 0 \\
0 & \lambda_{2}^{2}(s) & 0 \\
0 & 0 & \lambda_{3}^{2}(s)
\end{array}\right)
$$

As is shown in [9], the set of orthogonal transformations which satisfy (24) when $\mathbf{C}(s)$ is given by (31) form the group which represents orthotropy. If $D_{\text {orth }}$ represents this group of transformations, then

$$
g\left(t_{f}\right) \supseteq D_{\text {orth }} .
$$

For more information and details about deformation induced symmetries in first gradient models see [10].

\section{MATERIAL SPECIFIC RESULTS}

Other results can be obtained for the symmetry of the post-crystallization elastic response when the constitutive equation has a particular form. In order to illustrate the nature of such results, consider a simplification of constitutive equation (17) in which $w_{2}=w_{3}=w_{5}=\cdots=$ $w_{9}=0$, and $w_{1}$ and $w_{4}$ depend only on the scalar invariants of $\mathbf{B}(s)$. In this case, the constitutive equation, given by (19), after the completion of crystallization becomes

$$
\mathbf{T}(t)=b(t)\left[p_{0} \mathbf{I}+p_{1} \mathbf{B}(t)+p_{2} \mathbf{B}^{2}(t)\right]+\alpha \mathbf{I}+\mathbf{F}(t) \mathbf{A} \mathbf{F}^{T}(t),
$$

where $\alpha$ and $\mathbf{A}$ are constants defined by

$$
\alpha=\int_{t_{s}}^{t_{f}} a(s) w_{1} \mathrm{~d} s
$$


and

$$
\mathbf{A}=\int_{t_{s}}^{t_{f}} a(s) w_{4} \mathbf{C}(s)^{-1} \mathrm{~d} s
$$

The set of transformations $\mathbf{H}$ which satisfy (21) contains every orthogonal $\mathbf{H}$ which satisfies the condition

$$
\mathbf{H}^{T} \mathbf{A H}=\mathbf{A}
$$

Therefore,

$$
g\left(t_{f}\right) \supseteq\left\{\mathbf{H} \mid \mathbf{H} \in \mathcal{O} \text { and } \mathbf{H}^{T} \mathbf{A H}=\mathbf{A}\right\} .
$$

Since $\mathbf{C}(s)$ is symmetric, $\mathbf{A}$ is also symmetric and can be written in the form

$$
\mathbf{A}=\mathbf{P} \mathbf{A}_{d} \mathbf{P}^{T}
$$

in which $\mathbf{P}$ is orthogonal and

$$
\mathbf{A}_{d}=\left(\begin{array}{ccc}
a_{1} & 0 & 0 \\
0 & a_{2} & 0 \\
0 & 0 & a_{3}
\end{array}\right) .
$$

As shown in [9], the solution to (35) is given as follows:

(1) $g\left(t_{f}\right) \supseteq \mathcal{O}$ if $a_{1}=a_{2}=a_{3}$ :

(2) $g\left(t_{f}\right) \supseteq \mathbf{P} D_{\text {tran }} \mathbf{P}^{T}$ if $a_{1}=a_{2} \neq a_{3}$ :

(3) $g\left(t_{f}\right) \supseteq \mathbf{P} D_{\text {orth }} \mathbf{P}^{T}$ if $a_{1} \neq a_{2} \neq a_{3} \neq a_{1}$.

The preferred directions of symmetry are given by the eigenvectors of $\mathbf{A}$ (i.e. by $\mathbf{P}$ ). Thus, for this specific form of constitutive equation, we have shown that regardless of the deformation history during the time interval of crystallization, the post-crystallization response will have only one of the three symmetries listed above.

\section{CONCLUDING REMARKS}

We have developed a model which attempts to characterize some of the special characteristics of continuous phase transition as would be seen, for example, in polymer crystallization. The model is based, to some extent, on the microstructural changes and macroscopic responses seen in polymer crystallization, particularly in Natural Rubber. The model assumes that the response before the start of crystallization is elastic. The response after the completion of crystallization will also be elastic. The model is fully nonlinear, as would be required for polymers, and represents the proper starting point for the development of linearized versions.

The current model is developed for isothermal phase transitions. An extension to nonisothermal response will require a better understanding of the interaction between temperature, strain, and crystallization.

Finally, this model can be used in conjunction with the appropriate field equations in formulating initial value and boundary value problems. With it, one can follow the behavior of materials through the crystallization process for complex flow problems and, for example, calculate residual symmetry properties, residual stresses and permanent deformations.

\section{REFERENCES}

[1] L. MANDELKERN, Crystallization of Polymers. McGraw-Hill, New York (1964)

[2] L. R. G. Treloar, The Physics of Rubber Elasticity. Clarendon Press, Oxford (1975).

[3] A. PETERLIN, Crystallization phenomena. In Flow-Induced Crystallization in Polymer Systems (Edited by R. L. MILLER), pp. 1-29. Gordon \& Breach, London (1979).

[4] A. STEVENSON, J. Polym. Sci.: Polym. Phys. Edn 21, 553-572 (1983). 
[5] I. M. WARD, Mechanical Properties of Solid Polymers, 2nd edn. Wiley, New York (1983).

[6] A. N. GENT, Trans. Faraday Soc. 50, 521-533 (1954).

[7] R. L. MILLER, Status of the analysis of crystallization kinetic data. In Flow-Induced Crystallization in Polymer Systems (Edited by R. L. MILLER), pp. 31-56. Gordon \& Breach, London (1979).

[8] R. S. RIVLIN and J. L. ERICKSON, Rat. Mech. Anal. 4(2), (March 1955).

[9] M. NEGAHBAN and A. S. WINEMAN, Int. J. Nonlinear Mech. 24(6), 521-536 (1989),

[10] M. NEGAHBAN and A. S. WINEMAN, Int. J. Nonlinear Mech. 24(6), 537-549 (1989).

[11] M. NEGAHBAN, Material symmetry and the evolution of anisotropies in first gradient theories of nonlinear material behavior; inelasticity; polymer crystallization. Ph.D. dissertation, University of Michigan (August 1988).

\section{APPENDIX A}

\section{Two Alternate Forms of the Model}

Since $\mathbf{F}_{s}(t)=\mathbf{F}(t) \mathbf{F}^{-1}(s)$, the two variables $\mathbf{F}(t)$ and $\mathbf{F}_{s}(t)$ in the expression for the stress in the crystal can be replaced by either the set $\mathbf{F}(t)$ and $\mathbf{F}(s)$ or the set $\mathbf{F}_{s}(t)$ and $\mathbf{F}(s)$.

Selecting the variable set $\mathbf{F}(t)$ and $\mathbf{F}(s)$ results in a constitutive equation for an initially isotropic material given by

$$
\begin{aligned}
\mathbf{T}(t)= & b(t)\left[p_{0} \mathbf{I}+p_{1} \mathbf{B}(t)+p_{2} \mathbf{B}^{2}(t)\right]+\mathbf{F}(t) \int_{t_{s}}^{t} a(s)\left\{w_{1} \mathbf{I}+w_{2} \mathbf{C}(t)+w_{3} \mathbf{C}^{2}(t)+w_{4} \mathbf{C}(s)\right. \\
& +w_{5} \mathbf{C}^{\prime}(s)+w_{6}[\mathbf{C}(t) \mathbf{C}(s)+\mathbf{C}(s) \mathbf{C}(t)]+w_{7}\left[\mathbf{C}^{2}(t) \mathbf{C}(s)+\mathbf{C}(s) \mathbf{C}^{2}(t)\right] \\
& \left.+w_{8}\left[\mathbf{C}(t) \mathbf{C}^{2}(s)+\mathbf{C}^{2}(s) \mathbf{C}(t)\right]+w_{9}\left[\mathbf{C}^{2}(t) \mathbf{C}^{2}(s)+\mathbf{C}^{2}(s) \mathbf{C}^{2}(t)\right]\right\} \mathrm{d} s \mathbf{F}^{T}(t)
\end{aligned}
$$

where $p_{0}, p_{1}$, and $p_{2}$ are functions of the three isotropic invarients of $\mathbf{B}(t)$, and $w_{1}, \ldots, w_{y}$ are functions of the ten isotropic invarients of $\mathbf{C}(t)$ and $\mathbf{C}(s)$.

Selecting the variable set $\mathbf{F}_{s}(t)$ and $\mathbf{F}(s)$ result in a complicated form which we will not present.

\section{APPENDIX B}

\section{A Model Based on Volume Ratios}

A similar model for the stress can be developed based on assuming each portion of the material contributes to the value of the Cauchy Stress in proportion to its current value of volume ratio. In this case, one must provide a constitutive equation which allows the calculation of the current value of volume for each of the different phases. What follows is one such example.

Let $b(t)$ be the current value of mass ratio of amorphous material, and let $a(s)$ be the rate of transformation of the mass from one phase to the other (same as previously defined). Assume the value of crystal density at the time of formation of the crystal to be a known constant $\rho_{c 0}$. The volume of the crystal created in the interval ds is given by

$$
\mathrm{d} V_{c_{0}}=\frac{\mathrm{d} m_{c}}{\rho_{c_{0}}}=\frac{m_{0}}{\rho_{c_{0}}} a(s) \mathrm{d} s,
$$

where $\mathrm{d} V_{c_{0}}$ is the volume of the crystal at the time of its creation, $\mathrm{d} m_{c}$ is the mass of material transformed in the time interval $\mathrm{d} s$, and $m_{0}$ is the total mass of material.

Next we need to make an assumption on how much the individual constituents change in volume ratio, given the total change in volume ratio. If we assume that each constituent has a volume ratio change identical to the total material, we will have

$$
\mathrm{d} V_{c}=\frac{J(t)}{J(s)} \mathrm{d} V_{e_{0}}=m_{0} \frac{J(t) a(s)}{J(s) \rho_{c_{0}}} \mathrm{~d} s
$$

where $\mathrm{d} V_{c}$ is the current value of the volume of the crystal created in the interval $\mathrm{d} s, J(t)$ and $J(s)$ are the total volume ratio of the material at time $t$ and $s$, respectively. This results in the expression for the current value of the volume ratio of the crystal created in the interval $\mathrm{d} s$ as

$$
\frac{\mathrm{d} V_{c}}{V(t)}=\frac{\rho_{0} a(s)}{J(s) \rho_{c_{0}}} \mathrm{~d} s
$$

where $V(t)$ is the total volume of the material of mass $m_{0}$ at the current time and $\rho_{0}$ is the initial density of the untransformed material.

The constitutive equation for such a material would be given by

where

$$
\mathbf{T}(t)=\hat{b}(t) \mathbf{T}^{A}(t)+\int_{t_{s}}^{t} \frac{\rho_{\mathrm{g}} a(s)}{J(s) \rho_{c_{0}}} \mathbf{T}^{C}(t, s) \mathrm{d} s_{,}
$$

$$
\hat{b}(t)=1-\int_{t_{s}}^{t} \frac{\rho_{0} a(s)}{J(s) \rho_{c_{0}}} \mathrm{~d} s
$$

Since in a polymer like natural rubber the total change in volume ratio and density are in the order of several percent compared to several magnitudes of change in the value of the modulus, it seems justified to ignore these small effects. As a result, it seems justified to use a mass ratio based theory, like presented in the main text, as opposed to a volume ratio based theory, like presented in this section.

Other equations can be developed based on how the volume change is distributed between the different phases of the material. 